\title{
Ultrafast light-induced magnetization dynamics in ferromagnetic semiconductors
}

\author{
J. Chovan, E. G. Kavousanaki, and I. E. Perakis \\ Institute of Electronic Structure \& Laser, Foundation for Research and \\ Technology-Hellas and Department of Physics, University of Crete, Heraklion, Greece
}

(Dated: July 17, 2018)

\begin{abstract}
We develop a theory of the magnetization dynamics triggered by ultrafast optical excitation of ferromagnetic semiconductors. We describe the effects of the strong carrier spin relaxation on the nonlinear optical response by using the Lindblad semigroup method. We demonstrate magnetization control during femtosecond timescales via the interplay between circularly polarized optical excitation, hole-spin damping, polarization dephasing, and the Mn-hole spin interactions. Our results show a light-induced magnetization precession and relaxation for the duration of the optical pulse.
\end{abstract}

PACS numbers: 78.47.+p, 78.20.Ls, 75.50.Pp, 72.25.Rb

With the discovery of III-Mn-V ferromagnetic semiconductors [1], one can envision a new class of multifunctional devices that combine information processing and storage on a single chip with low power consumption. One of the challenges facing such future devices concerns the speed of the basic processing unit, governed by the dynamics of the collective spin and its ability to respond quickly to time-dependent external fields. Ultrafast pump-probe magneto-optical spectroscopy has been used to study the magnetization dynamics triggered by femtosecond optical pulses in both metals $2,|3|$ and ferromagnetic semiconductors [4, 5, 6, 7]. Transient magnetic effects have been observed even in the initial nonthermal temporal regime, where the concept of carrier temperature is not meaningful [2, 8].

In III-Mn-V semiconductors, the ferromagnetic order is induced by the interaction between the itinerant valence band hole spins and the localized Mn spins [9, 10]. The magnetization is therefore sensitive to the itinerant carrier properties, which in III-V semiconductors can be well controlled with ultrashort optical pulses [11]. The combined magnetic and optical properties of this system open new possibilities for manipulating the collective spin without using magnetic fields. This can be achieved, e.g., by exciting spin-polarized carriers with circularly-polarized femtosecond optical pulses. The effects of such photoexcited spin on the ferromagnetic state can be studied by monitoring the change in the macroscopic magnetization as function of time using ultrafast spectroscopy [2, 3, 4, 5, 6, 7]. Light-induced magnetization and very fast $\mathrm{Mn}$ spin rotation towards the direction of propagation of the circularly polarized optical pulse, perpendicular to the ground state magnetization, were reported in $\mathrm{Ga}(\mathrm{Mn})$ As ferromagnetic epilayers 7 . Kimel et.al. 4 observed a photoinduced magnetization attributed to the photoexcited conduction electron spin. Kojima et.al. [6] interpeted their data in terms of a rapid (ps) rise in carrier temperature, followed by a slow rise in the spin temperature and a magnetization reduction over 100 s of ps. For very intense photoexcitation of $\operatorname{In}(\mathrm{Mn}) \mathrm{As}$, Wang et.al. [5] observed a fast (100s of fs) and a slow (100s of ps) demagnetization regime. Other possibilities for light-induced magnetic effects in non-magnetic semiconductors were recently explored theoretically [12, 13]. In doped semiconductors, a light-induced Kondo effect was predicted [12], while a light-induced paramagneticto-ferromagnetic phase transition was predicted in undoped semiconductors 13.

In this letter, we develop a theory of the ultrafast nonlinear optical dynamics and light-induced hole and Mn spin relaxation in ferromagnetic semiconductors. We treat the magnetic exchange interactions within the time-dependent Hartree-Fock approximation, and treat the hole spin damping and resulting nonlinear polarization dephasing within the Lindblad semigroup method 14]. We predict an ultrafast light-induced precession and relaxation of the collective magnetization towards a direction determined by the nonlinear optical polarizations. The above spin dynamics results from the interplay between the nonlinear optical excitation, the interaction between the photoexcited hole and Mn spins, and the strong hole-spin damping and optical polarization dephasing, The predicted dynamics is observable with ultrafast magneto-optical pump-probe spectroscopy.

We adopt the simple [9, 10] two-band sp-d model Hamiltonian $H(t)=K_{e}+K_{h}+H_{\text {exch }}+H_{L}(t)$, where $K_{h}$ $\left(K_{e}\right)$ is the kinetic energy of the valence band heavy holes (conduction band electrons) created by $\hat{h}_{\mathbf{k} \sigma}^{\dagger}\left(\hat{e}_{\mathbf{k} \sigma}^{\dagger}\right)$ with total angular momentum $J_{z}= \pm 3 / 2(\operatorname{spin} \sigma= \pm 1 / 2)$ and dispersion $\varepsilon_{\mathbf{k}}^{v}=k^{2} / 2 m_{h}\left(\varepsilon_{\mathbf{k}}^{c}=k^{2} / 2 m_{e}\right)$ (we set $\hbar=1)$. Unlike in II-VI semiconductors, the Mn impurities provide both a hole Fermi sea and randomly distributed $S=5 / 2$ localized spins, $\mathbf{S}_{j}$, located at positions $\mathbf{R}_{j}$. These local spins interact strongly with the itinerant hole spins via the Kondo-like exchange interaction

$$
H_{\mathrm{exch}}=\frac{J}{V} \sum_{j \mathbf{k} \mathbf{k}^{\prime} \sigma \sigma^{\prime}}\left(\mathbf{S}_{j} \cdot \sigma\right)_{\sigma \sigma^{\prime}} e^{i\left(\mathbf{k}-\mathbf{k}^{\prime}\right) \cdot \mathbf{R}_{j}} \hat{h}_{\mathbf{k} \sigma}^{\dagger} \hat{h}_{\mathbf{k}^{\prime} \sigma^{\prime}}
$$

where $\sigma$ is the hole spin operator and $V$ is the volume. The coupling of right-circularly polarized optical fields is 
described in the rotating frame by 11$]$

$$
H_{L}(t)=-d(t) \sum_{\mathbf{k}} \hat{e}_{\mathbf{k} \downarrow}^{\dagger} \hat{h}_{-\mathbf{k} \uparrow}^{\dagger}+h . c,
$$

where $d(t)=\mu_{c v} \mathcal{E}(t)$ is the Rabi energy, $\mu_{c v}$ is the dipole transition matrix element, and $\mathcal{E}(t) \propto \exp \left[-t^{2} / \tau_{p}^{2}\right]$ is the optical pulse, with central frequency $\omega_{p}$ and duration $\tau_{p}$. In the above Hamiltonian, the spin quantization $\mathrm{z}^{-}$ axis was taken parallel to the direction of optical pulse propagation, which similar to Ref. 7] was chosen here perpendicular to the ground state spin polarization.

The ground state of the above Hamiltonian is typically described by treating the interaction Eq.(11) within the mean field virtual crystal approximation, which neglects spatial correlations and assumes uniformly distributed classical Mn spins [9, 10]. Within this approximation, the holes experience an effective magnetic field proportional to the average $\mathrm{Mn}$ spin [9, 10]. The valence states then split into two spin-polarized bands, separated by the magnetic exchange energy $J c S$, where $c$ is the $\mathrm{Mn}$ concentration, and populated by the hole Fermi sea. The spins of the above bands point antiparallel and parallel to the $\mathrm{Mn}$ spin, which is taken here to define the $\mathrm{x}$-axis.

We calculated the ultrafast nonlinear optical response by solving the equations of motion for the density matrix. For right-circularly polarized light, spin- $\uparrow$ electron states are not populated for the above Hamiltonian, and we only need to consider the interband optical polarizations $P_{\mathbf{k} \sigma}=\left\langle\hat{h}_{-\mathbf{k} \sigma} \hat{e}_{\mathbf{k} \downarrow}\right\rangle$, the carrier populations, the average $\mathbf{M n}$ spin $\mathbf{S}$, and the hole spin $\mathbf{s}_{\mathbf{k}}^{h}$, whose components are $s_{\mathbf{k} z}^{h}=\sum_{\sigma} \sigma\left\langle\hat{h}_{\mathbf{k} \sigma}^{\dagger} \hat{h}_{\mathbf{k} \sigma}\right\rangle$ and $s_{\mathbf{k}+}^{h}=\left\langle\hat{h}_{\mathbf{k} \uparrow}^{\dagger} \hat{h}_{\mathbf{k} \downarrow}\right\rangle$, where $s_{\mathbf{k}+}^{h}=s_{\mathbf{k} x}^{h}+i s_{\mathbf{k} y}^{h}$. By factorizing all higher density matrices, we obtain a closed system of equations analogous to the semiconductor Bloch equations 11] generalized to include the spin degrees of freedom. Due to the selection rules, Eq. (2), right-circularly polarized light excites directly the spin- $\uparrow$ hole-spin- $\downarrow$ electron polarization $P_{\uparrow}$ :

$$
\begin{aligned}
& \left(i \partial_{t}-\Omega_{\mathbf{k}}-\frac{J c S_{z}}{2}\right) P_{\mathbf{k} \uparrow}-\frac{J c S_{-}}{2} P_{\mathbf{k} \downarrow}= \\
& -d(t)\left[1-\left\langle\hat{e}_{\mathbf{k} \downarrow}^{\dagger} \hat{e}_{\mathbf{k} \downarrow}\right\rangle-\left\langle\hat{h}_{\mathbf{k} \uparrow}^{\dagger} \hat{h}_{\mathbf{k} \uparrow}\right\rangle\right]
\end{aligned}
$$

where $S_{ \pm}(t)=S_{x}(t) \pm i S_{y}(t)$ and $\Omega_{\mathbf{k}}=\varepsilon_{\mathbf{k}}^{v}+\varepsilon_{\mathbf{k}}^{c}-\omega_{p}-i / T_{2}$, where $T_{2}$ is the polarization dephasing time. The rhs of the above equation describes the Pauli-blocking nonlinearity (Phase Space Filling) 11], determined by the equations of motion for the carrier populations, with initial condition the Fermi-Dirac distribution of the hole Fermi sea. Additional nonlinearities come from the lightinduced changes in the $\mathrm{Mn}$ spin $\mathbf{S}(t)$ from its ground state configuration, discussed below, which result in a timedependent effective magnetic field $J_{c} \mathbf{S}(t)$. The latter gives a time-dependent e-h pair energy $\propto S_{z}(t)$, which renormalizes the spin-polarized hole bands, and a time- dependent coupling $\propto S_{-}(t)$ between $P_{\uparrow}$ and $P_{\downarrow}$ :

$$
\left(i \partial_{t}-\Omega_{\mathbf{k}}+\frac{J c S_{z}}{2}\right) P_{\mathbf{k} \downarrow}-\frac{J c S_{+}}{2} P_{\mathbf{k} \uparrow}=d(t) s_{\mathbf{k}+}^{h} .
$$

$P_{\downarrow}$ is also generated due to the hole spin coherence, photoexcited or ground state, described by $s_{+}^{h}$ (rhs of Eq.(4)). In turn, the hole and Mn spins depend on the optical polarizations and precess due to the exchange interaction as described by the equations of motion

$$
\partial_{t} s_{\mathbf{k}}^{h}=J c \mathbf{S} \times \mathbf{s}_{\mathbf{k}}^{h}+d(t) \mathbf{h}_{\mathbf{k}}, \partial_{t} \mathbf{S}=J \sum_{\mathbf{k}} \mathbf{s}_{\mathbf{k}}^{h} \times \mathbf{S} .(5)
$$

In the above equation, the photoexcited hole spin is determined by the nonlinear optical polarizations (and thus the interactions) as described by the vector

$$
\mathbf{h}_{\mathbf{k}}=\operatorname{Im}\left(\mathrm{P}_{\mathbf{k} \downarrow},-i \mathrm{P}_{\mathbf{k} \downarrow}, \mathrm{P}_{\mathbf{k} \uparrow}\right) .
$$

During the femtosecond time scales of interest here, we neglected for simplicity the much slower precession and relaxation of the Mn spin due to the effective magnetic field caused by the magnetic anisotropy and the Gilbert damping [15, 16]. The nonlinear effects were treated nonperturbatively by solving numerically the above system of coupled equations.

Relaxation in ferrmagnetic semiconductors mostly occurs during time scales longer than the pulse duration. A notable exception is the hole spin relaxation, which occurs within 10's of fs due to the strong spin-orbit coupling in the valence band and the disorder-induced scattering between the different momentum states [17]. Carrier spin relaxation is typically described within the spin Bloch equations by introducing relaxation terms so that the spin relaxes to a direction opposite to the Mnspin [15, 17]. However, this spin relaxation also leads to strong dephasing of the optical polarizations and coupling between $P_{\uparrow}$ and $P_{\downarrow}$. Thus, the dephasing of both the spin and the optical polarizations must be treated consistently, including the light-induced nonlinear contributions. We treated all the above effects by using the Lindblad semigroup description of dissipative quantum dynamics [14]. Under the general assumptions of linear coupling between bath and system operators $L_{\mathbf{k}}$ and positivity and semigroup-type density matrix time evolution, the relaxation contribution is given by [14]

$$
\left.\partial_{t} \rho\right|_{\mathrm{rel}}=\sum_{\mathbf{k k}^{\prime}} \frac{\Gamma_{\mathbf{k} \mathbf{k}^{\prime}}}{2}\left\langle 2 L_{\mathbf{k}} \hat{\rho} L_{\mathbf{k}^{\prime}}^{\dagger}-L_{\mathbf{k}^{\prime}}^{\dagger} L_{\mathbf{k}} \hat{\rho}-\hat{\rho} L_{\mathbf{k}^{\prime}}^{\dagger} L_{\mathbf{k}}\right\rangle(7)
$$

The Lindblad operators $L_{\mathbf{k}}=\hat{h}_{\mathbf{k} \Downarrow}^{\dagger} \hat{h}_{\mathbf{k} \Uparrow}$, where $\hat{h}_{\mathbf{k} \Uparrow}^{\dagger}\left(\hat{h}_{\mathbf{k} \Downarrow}^{\dagger}\right)$ creates a hole with spin parallel (anti-parallel) to the Mn spin, describe relaxation of the hole spin toward the direction opposite to $\mathbf{S}(t)$ with a rate $\Gamma_{\mathbf{k k}^{\prime}}=\delta_{\mathbf{k k}^{\prime}} \Gamma_{s}$. The detailed equations, obtained from Eq. (7) by factorizing the higher density matrices, will be presented 


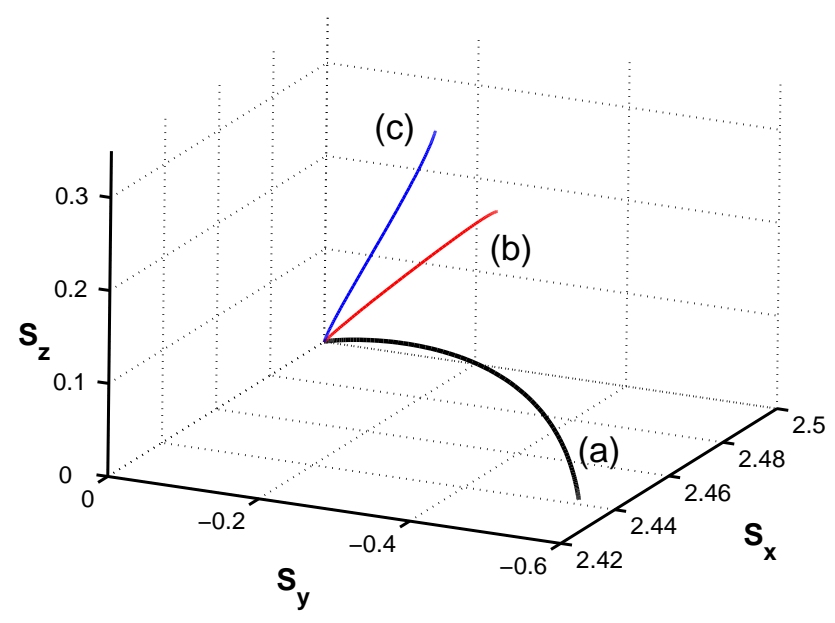

FIG. 1: Mn-spin trajectory for three different relaxation regimes: (a) $T_{2}=2 T_{1}=330 \mathrm{fs}, \Gamma_{s}=0$, (b) $T_{2}=2 T_{1}=330 \mathrm{fs}$, $1 / \Gamma_{s}=10.5 \mathrm{fs}$, (c) $T_{1}=165 \mathrm{fs}, T_{2}=1 / \Gamma_{s}=10.5 \mathrm{fs}$.

elsewhere. Here we note that the hole spin component perpendicular to $\mathbf{S}$ dephases with a rate $\Gamma_{s}$, while the spin component parallel to $\mathbf{S}$ relaxes with a rate $2 \Gamma_{s}$ towards $-s_{\max }+s_{\max }^{2}-\left(\mathbf{s}_{\mathbf{k}}^{h}\right)^{2}$, where $s_{\max }$, with $s_{\max }-s_{\max }^{2}=n_{\mathbf{k}}^{h} / 2-\left(n_{\mathbf{k}}^{h} / 2\right)^{2}$, is the maximum hole spin for given number of holes $n_{\mathbf{k}}^{h}=\sum_{\sigma}\left\langle\hat{h}_{\mathbf{k} \sigma}^{\dagger} \hat{h}_{\mathbf{k} \sigma}\right\rangle$. Eq.(7) also gives optical polarization dephasing and coupling between $P_{\uparrow}$ and $P_{\downarrow}$ that depend on $\mathbf{S}(t), \mathbf{s}^{h}(t)$, and the hole populations, whose expressions will be given elsewhere. We note that electron and Mn spin relaxation can also be included by using analogous Lindblad operators.

Fig. 1 shows the light-induced time evolution of the Mn spin for pulse duration $\tau_{p}=250 \mathrm{fs}$, Rabi energy d $=60 \mathrm{meV}$, and detuning $10 \mathrm{meV}$ below the threshold of interband absorption. Other parameters used in our calculation are the exchange energy $J c S=125 \mathrm{meV}$, hole Fermi energy $100 \mathrm{meV}$, fraction of initial holes 0.33 of the Mn impurities, and $m_{h} / m_{e}=7.15$. To elucidate the role of relaxation, we compare in Fig. 1 the Mn spin trajectories for different values of $\Gamma_{s}$ and $T_{2}$. The Mn spin rotates in a clockwise direction within the $\mathrm{x}-\mathrm{y}$ plane perpendicular to the direction of optical pulse propagation. With increasing hole spin damping $\Gamma_{s}$, it develops a large out of plane $\mathrm{z}$-component that increases further with decreasing $T_{2}$.

The spin dynamics of Fig.1 comes from the Mn spin precession around the mean hole spin. Unlike for a magnetic field, the latter changes strongly with time, as determined by the nonlinear optical polarizations, the spin relaxation, and the precession around $\mathbf{S}$ (Eq.(5)). For the parameters in Fig.1, the hole spin follows overall the Mn spin trajectory, with the exception of a component perpendicular to $\mathbf{S}$, determined by the nonlinear optical polarizations and the spin relaxation, which triggers the Mn spin dynamics. The light-induced deviations from

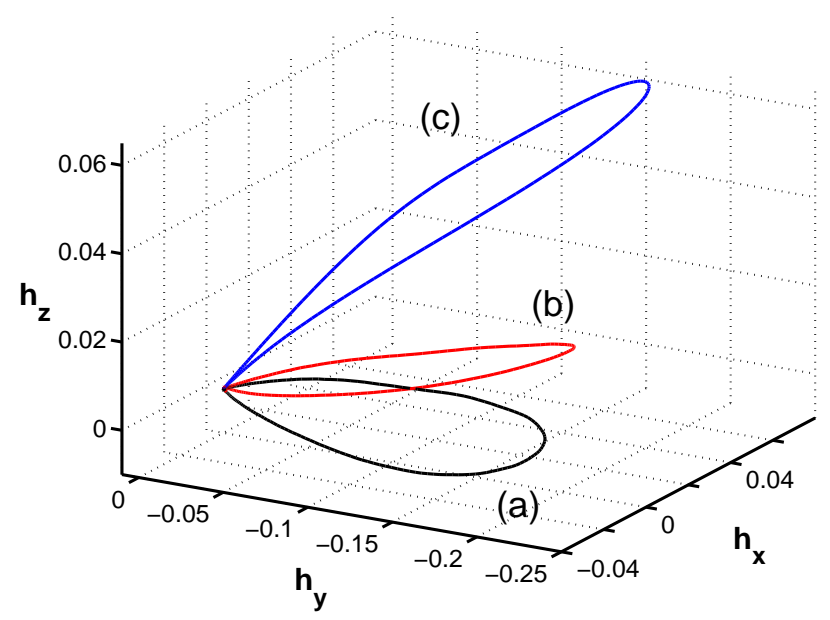

FIG. 2: Trajectories of the light-induced field $\mathbf{h}$ (units of $\left.d(0) / 2 E_{F}\right)$ for the three relaxation regimes of Fig. 1.

the equilibrium spin configurations occur within the pulse duration and increase with its duration and intensity.

At first glance, the clockwise $\mathrm{Mn}$ spin rotation is suprising given that, due to the selection rules Eq.(2), right-circularly polarized light creates spin-up holes that should lead to counter-clockwise rotation (see Eq.(15)). In fact, our numerical results show that the $\mathrm{z}$-component of the photoexcited hole spin is negative due to the exchange interaction. This is a consequence of the fact that $\mathbf{s}^{h}$ is photoexcited via the nonlinear optical polarizations and not by the optical field directly (see Eq.(50). Also striking is the strong effect of the hole spin relaxation on the Mn spin trajectory: it leads to the development, within the pulse duration, of a large component $S_{z}$ in the direction of optical pulse propagation despite the absence of any Mn spin damping in Eq.(15). A very fast Mn spin tilt toward the $\mathrm{z}$-direction was observed experimentally [], however for weaker photoexcitation. The spin dynamics in Fig.1 is significant for Rabi energies larger than a few tens of meVs, which corresponds to stronger photoexcitation than in Ref. [4 but not as strong as in Ref. [5], where heating effects [2] become pronounced.

To interpret the above results, we consider the stationary (adiabatic following) limit, where the hole spin precesses and/or relaxes much faster than the pulse duration and period of $\mathrm{Mn}$ spin precession, and can thus adjust to the instantaneous $d(t)$ and $\mathbf{S}(t)$ values. Neglecting the component of $\partial_{t} \mathbf{s}^{h}$ perpendicular to $\mathbf{S}$, we can then eliminate the hole spin from Eq. (5) and obtain after some algebra and noting that $\mathbf{S} \cdot \partial_{t} \mathbf{S}=0$

$$
\partial_{t} \mathbf{S}=\frac{J d(t)}{(J c S)^{2}+\Gamma_{s}^{2}}\left[\Gamma_{s} \mathbf{h} \times \mathbf{S}-J c \mathbf{S} \times(\mathbf{S} \times \mathbf{h})\right]
$$

where $\mathbf{h}(t)=\sum_{\mathbf{k}} h_{\mathbf{k}}$ is an effective magnetic field determined by the nonlinear optical polarizations (Eq.(66)). The above equation for the Mn spin demonstrates a lightinduced precession around $\mathbf{h}(t)$ and Gilbert damping-like 
relaxation of $\mathbf{S}$ toward $\mathbf{h}(t)$. Such ultrafast nonlinear optical effects should be contrasted to the static effects governed by the magnetic anisotropy 15,16 .

The light-induced effective magnetic field $\mathbf{h}(t)$, Eq. (6) that governs the Mn spin dynamics has z-component determined by the dissipative part of the nonlinear optical polarization, $\operatorname{Im} P_{\uparrow}$, and in-plane components determined by $P_{\downarrow}$. Its time-dependence is shown in Fig.2. The exchange interaction plays an important role in determining $\mathbf{h}(t)$. To see this, we note that, for $J=0, P_{\downarrow}=0$. The corresponding $\mathbf{h}$ then points along the $\mathbf{z}^{-}$axis, with magnitude determined by the dephasing of $P_{\uparrow}$ and, for $T_{2}<\tau_{p}$, proportional to the optical pump rate. In this case, the Mn spin would relax to the $\mathrm{z}$-axis. However, the exchange interaction and spin relaxation drastically affect the optical polarizations and thus $\mathbf{h}(t)$. For example, by coupling the hole spin- $\uparrow$ and spin $-\downarrow$ states, they lead to a large $P_{\downarrow}$ and thus a large x-y plane component of the effective magnetic field $\mathbf{h}$. This is demonstrated in Fig.2, which shows $\mathbf{h}(t)$ for different values of the relaxation rates. In fact, for sufficiently long $T_{2}$ and detunings close to or below the interband absorption threshold, $\operatorname{Re} P_{\downarrow}$ exceeds $\operatorname{Im} P_{\uparrow}$, in which case the light-induced magnetic field $\mathbf{h}$ points mostly along the $\mathrm{x}-\mathrm{y}$ plane, in a direction almost perpendicular to the ground state magnetization (x-axis) (see Fig.2(a)). For $\Gamma_{s}=0$, the second term on the rhs of Eq. (8) then leads to Mn spin relaxation mostly within the $\mathrm{x}-\mathrm{y}$ plane, while with increasing $\Gamma_{s}$, the first term leads to precession out of the $\mathrm{x}-\mathrm{y}$ plane, consistent with the numerical results of Fig.1. The polarization dephasing, due to the hole spin damping as well as the disorder and other contributions, enhances the relative magnitude of $\operatorname{Im} \mathbf{P}_{\uparrow}$ and thus $h_{z}$ (see Figs.2(b) and 2(c)). As a result of $h_{z}, \mathbf{S}$ develops an additional z-component as it relaxes toward $\mathbf{h}$. One should note that the rhs of Eq. (8) vanishes after the optical pulse is gone and thus the light-induced Mn spin relaxation and precession only occurs during the pulse. Furthermore, the optical polarizations, and therefore $\mathbf{h}(t)$, depend on the spins, and thus the Mn spin dynamics leads to an additional timedependence of $\mathbf{h}(t)$ and to corresponding nonlinearities. This can be seen in Fig.2, where the trajectories of $\mathbf{h}(t)$ have a loop-like shape. Finally, the Phase Space Filling optical nonlinearity also affects the time-dependence of $\mathbf{h}(t)$, Eq.(6), and thus the Mn spin relaxation Eq. (8).

To gain insight into the effects of the optical excitation and exchange interaction on the hole spin dynamics, one can solve analytically the polarization equations of motion, Eqs. (3) and (4), for $T_{2}<\tau_{p}$ in the stationary limit, discussed elsewhere. For example, we obtain this way for the $\mathrm{x}-\mathrm{y}$ spin components $\left(\Gamma_{s}=0\right)$

$$
\begin{aligned}
& \partial_{t} s_{\mathbf{k}+}^{h}=J\left[1+\frac{d^{2}(t) / 2}{\Omega_{\mathbf{k}}^{2}-(J c S / 2)^{2}}\right] \mathbf{S} \times\left.\mathbf{s}_{\mathbf{k}}^{h}\right|_{+} \\
& +\frac{i d^{2}(t) \Omega_{\mathbf{k}}}{\Omega_{\mathbf{k}}^{2}-(J c S / 2)^{2}}\left[s_{\mathbf{k}+}^{h}+\frac{J\left(1-n_{\mathbf{k}} / 2\right)}{2 \Omega_{\mathbf{k}}} S_{+}\right]
\end{aligned}
$$

where $n_{\mathbf{k}}=\sum_{\sigma}\left\langle\hat{h}_{\mathbf{k} \sigma}^{\dagger} \hat{h}_{\mathbf{k} \sigma}\right\rangle$. The first term on the rhs describes hole spin precession around $\mathbf{S}(t)$ triggered by a time-dependent effective exchange interaction. The second term describes a light-induced in-plane rotation of the hole spin, even for $S_{z}=0$, while its dissipative part describes a light-induced hole spin relaxation. Finally, we note that, for very strong photoexcitation 5 and/or detunings well above the absorption edge, the heating of the hole Fermi sea can become significant. Similar to metals [2], such hot hole effects can be treated by introducing a time-dependent effective temperature.

In conclusion, we presented a theory of ultrafast nonlinear optical and spin dynamics in $\operatorname{III}(\mathrm{Mn}) \mathrm{V}$ ferromagnetic semiconductors. We demonstrated a light-induced Mn spin precession and relaxation, which can lead to a magnetization tilt of several tens of degrees. We showed that the latter is determined by the nonlinear optical polarizations and depends critically on the exchange interactions, dephasing, and hole spin relaxation. The nonlinear light-induced magnetic effects discussed here occur during the optical pulse and can be observed with ultrafast pump-probe magneto-optical spectroscopy.

This work was supported by the EU Research Training Network HYTEC (HPRN-CT-2002-00315).

[1] H. Ohno, Science 281, 951 (1998).

[2] L. Guidoni et. al., Phys. Rev. Lett. 89, 017401 (2002); E. Beaurepaire et. al., 76, 4250 (1996); J.-Y. Bigot et. al., Phys. Rev. Lett. 93, 077401 (2004).

[3] B. Koopmans et. al., Phys. Rev. Lett. 85, 844 (2000); J. Phys. Condens. Matter 15, S723 (2003).

[4] A. V. Kimel et. al., Phys. Rev. Lett. 92, 237203 (2004).

[5] J. Wang et. al., cond-mat/0504611 (2005).

[6] E. Kojima et. al., Phys. Rev. B 68, 193203 (2003).

[7] A. Oiwa et. al., Phys. Rev. Lett. 88, 137202 (2002); Y. Mitsumori et. al., Phys. Rev. B 69, 033203 (2004).

[8] R. Gomez-Abal et. al., Phys. Rev. Lett. 92, 27402 (2004); G. P. Zhang and W. Hübner, Phys. Rev. Lett. 85, 3025 (2000); Phys. Rev B 58, R5920 (1998).

[9] T. Dietl et. al., Science 287, 1019 (2000); T. Jungrith et. al., cond-mat/0505215 (2005).

[10] See e.g. J. König et. al. in Electronic Structure and Magnetism of Complex Materials, eds. J. Singh and D. A. Papaconstantopoulos (Springer-Verlag, Berlin, 2003).

[11] See e.g. W. Schäfer and M. Wegener, Semiconductor Optics and Transport Phenomena (Springer-Verlag, Berlin, 2002); N. Linder and L. J. Sham, Physica E 2, 412 (1998).

[12] T. V. Shahbazyan, I. E. Perakis, and M. E. Raikh, Phys. Rev. Lett. 84, 5896 (2000).

[13] J. Fernández-Rossier et. al., Phys. Rev. Lett. 93, 127201 ( 2004).

[14] See e.g. G. Lindblad, Commun. Math. Phys. 48, 119 (1976); R. Kosloff and S. A. Rice, J. Chem. Phys. 72, 4591 (1980).

[15] Y. Tserkovnyak, G. A. Fiete, and B. I. Halperin, Appl. Phys. Lett. 84, 5234 (2004).

[16] A. H. Mitchell, Phys. Rev. 105, 1439 (1957); J. Sinova 
et. al., Phys. Rev. B 69, 085209 (2004); J. FernándezRossier et. al., cond-mat/0304492

76, 323 (2004).

[17] I. Zutic, J. Fabian, and S. Das Sarma, Rev. Mod. Phys. 